\title{
Role of vacancies in metal-insulator transitions of crystalline phase-change materials
}

\author{
W. Zhang ${ }^{1}$, A. Thiess ${ }^{2}$, P. Zalden ${ }^{3}$, R. Zeller ${ }^{2}$, P. H. Dederichs ${ }^{2}$, \\ J.-Y. Raty ${ }^{4}$, M. Wuttig ${ }^{3,5}$ * S. Blügel ${ }^{2,5}$, and R. Mazzarello ${ }^{1,5 \dagger}$ \\ 1 Institut für Theoretische Festkörperphysik, RWTH Aachen University, D-52056 Aachen, Germany \\ 2 Peter Grünberg Institut and Institute for Advanced Simulations, \\ Forschungszentrum Jülich, D-52425 Jülich, Germany \\ 3 I. Physikalisches Institut (IA), RWTH Aachen University, D-52056 Aachen, Germany \\ ${ }^{4}$ Physique de la Matière Condensée, B5, Université de Liège, B4000 Sart-Tilman, Belgium \\ 5 JARA-Fundamentals of Future Information Technology, \\ RWTH Aachen University, D-52056 Aachen, Germany
}

(Dated: July 25, 2012)

\begin{abstract}
The study of metal-insulator transitions in crystalline solids is a subject of paramount importance, both from the fundamental point of view and for its relevance to the transport properties of materials. Recently, a metal-insulator transition governed by disorder was observed in crystalline phase-change materials. Here we report on calculations employing Density Functional Theory, which identify the microscopic mechanism that localizes the wave functions and is driving this transition. We show that, in the insulating phase, the electronic states responsible for charge transport are localized inside regions having large vacancy concentrations. The transition to the metallic state is driven by the dissolution of these vacancy clusters and the formation of ordered vacancy layers. These results provide important insights on controlling the wave function localization, which should help to develop conceptually new devices based on multiple resistance states.
\end{abstract}

Understanding and tailoring charge transport in crystalline solids is a very important aspect of solid state physics, also in view of its relevance to a vast number of applications. Based upon the value of the resistivity in the limit of zero temperature, two different classes of solids can be distinguished: while metals have a finite resistivity, the resistivity of insulators diverges. It is particularly interesting to study compounds which undergo a metal insulator transition (MIT) due to charge carrier localization. Two different mechanisms have been suggested to explain such electronically driven MITs. Anderson [1] showed that strong disorder can localize electronic states at the Fermi energy, leading to insulating behaviour. Mott [2], on the contrary, emphasized the role of correlations for charge carrier localization.

Conceptually these two transitions are very different; experimentally, however, they are quite difficult to separate. This can be seen for crystalline semiconductors, in particular doped silicon [3-5], the best studied case of a system exhibiting an electronically driven MIT. It is now well established that both disorder and correlations play an important role in these systems. In fact, until very recently, there was to our knowledge no single class of crystalline solids where the MIT was exclusively governed by disorder. Hence, one can wonder if theoretical studies would be able to elucidate the origin of an MIT in a real solid. Indeed, a large number of model systems have been developed and investigated, with the aim of describing the essential physics of Anderson [6-10] and Mott [11-13] type MITs. To study real solids, however, it is mandatory to accurately describe the structural properties and the electronic wavefunctions. Employing density functional theory (DFT) calculations hence could be very rewarding. However, in general, modeling disorder to describe the MIT requires large system sizes, which are still difficult to handle with DFT codes. For phosphorous doped silicon (Si:P), for example, the critical carrier concentration $\mathrm{n}_{c}$ at the MIT is $3.8 \cdot 10^{18} \mathrm{~cm}^{3}$ [4]. If each donor atom would be ionized, 1 in every $12.500 \mathrm{Si}$ atoms would have to be replaced by a phosphorous donor. One would presumably need at least 10 dopant atoms and hence a system size of 125.000 atoms to catch a glimpse of the driving force for the MIT in Si:P. Clearly, such a system size is beyond the range of present DFT codes. For some systems, such as amorphous silicon, tight-binding Hamiltonians were used to investigate Anderson localization in large models containing several thousands of atoms [14].

Recently, compelling evidence for disorder induced localization has been observed in the crystalline phasechange material (PCM) $\mathrm{GeSb}_{2} \mathrm{Te}_{4}$ (GST) [15], where the measured carrier concentration was $2 \cdot 10^{20} \mathrm{~cm}^{3}$, corresponding to one charge carrier per 150 atoms. Therefore, we can expect to obtain important insights on the origin of this MIT by considering computationally feasible system sizes of one to several thousand atoms. Furthermore, while in experimental studies of semiconductors the MIT is usually induced by doping, which affects both the electron correlation as well as the disorder, in GST the MIT occurs at fixed stoichiometry. DFT now offers the unique opportunity to study a material with the same stoichiometry but different degrees of order. This opens up the possibility to identify the microscopic origin of localization in crystalline PCMs within a fully $a b$ initio approach.

Experimentally, it has been observed that in crystalline GST the resistivity at $4 \mathrm{~K}$ changed by more than 6 orders of magnitude upon increasing annealing temperature [15]. While the GST films heated to temperatures below $250{ }^{\circ} \mathrm{C}$ showed a negative change of resistance with 
temperature (TCR) and a diverging resistivity in the zero temperature limit, samples annealed above $250{ }^{\circ} \mathrm{C}$ led to films with a much lower (finite) resistance and a positive TCR, i.e. metallic behaviour. This transition from an insulating to a metallic state has been attributed to an increasing order in the crystalline phase. Correlation effects could be shown to be insignificant to explain this MIT [15]. This property was related to the unique bonding of the $p$ electrons in crystalline PCMs ("resonance bonding"), which leads to a high static dielectric constant and thus very weak effective electron interactions [16, 17]. On the other hand, PCMs such as GST are characterized by a high concentration of randomly arranged vacancies on the Ge/Sb sublattice ( $25 \%$ in GST), as well as a statistical distribution of $\mathrm{Ge}$ and $\mathrm{Sb}$ atoms on this sublattice. Furthermore, there are significant atomic displacements away from the sites of a perfect lattice (Peierls distortions $[18,19])$. This affects the overlap between adjacent $p$ orbitals, which impacts the electronic properties.

All of these different effects contribute to the pronounced disorder and make it plausible that charge carriers can indeed be localized. However, the dominant effect responsible for Anderson localization has not yet been identified. This issue is important not only from a fundamental point of view, but also in the fascinating perspective of building multiple-resistance devices by changing the amount of disorder in GST in a controlled manner. This technology would offer a dramatic increase in data density with respect to current non-volatile phase-change memories based on PCMs [20], which exploit the strong resistivity contrast between their cubic and amorphous phase. With these goals in mind, we have performed DFT computations which pinpoint the microscopic mechanism responsible for charge carrier localization in crystalline PCMs. To our knowledge, this is the first time that DFT has been successfully employed to identify the microscopic origin of an MIT in a disordered solid.

As-deposited amorphous GST crystallizes into a cubic phase (c-GST) or a hexagonal phase (h-GST) at low and high annealing temperatures respectively [15]. To shed light on the electronic properties of the two phases, a number of large supercells (containing up to 3584 atoms) of c-GST and h-GST with different degrees of disorder were generated and investigated by DFT. Disorder was varied by progressively reducing the randomness in the distribution of vacancies, $\mathrm{Ge}$ and $\mathrm{Sb}$ atoms. Two complementary electronic-structure methods were used: the Korringa-Kohn-Rostoker (KKR) Green function method $[21,22]$ and a wavefunction approach (see Methods).

Experimentally, the MIT was shown to occur in the hexagonal phase [15]. Nevertheless, we start our analysis discussing first the fully disordered cubic rock-salt phase, in that the study of this phase enables one to easily identify the structural configurations responsible for the localization of electrons in both phases. At anneal- ing temperatures between 150 and $200{ }^{\circ} \mathrm{C}$, amorphous GST crystallizes into this metastable structure [15]. This phase is also obtained in phase-change devices [23] upon fast recrystallization of the amorphous bits induced by heating. There is experimental evidence [24] that one sublattice is occupied by Te atoms only, whereas in the second one $25 \% \mathrm{Ge}, 50 \% \mathrm{Sb}$ and $25 \%$ vacancies are arranged in a random fashion. To describe this phase, we considered two supercells composed of $\mathrm{Ge}_{125} \mathrm{Sb}_{250} \mathrm{Te}_{500}$ and $\mathrm{Ge}_{512} \mathrm{Sb}_{1024} \mathrm{Te}_{2048}$, in which vacancies, Ge and $\mathrm{Sb}$ were placed on the second sublattice employing a random number generator. Hence, the obtained distribution of vacancies, Ge and Sb was completely uncorrelated. In the first step, relaxations from the ideal lattice positions were not included. $p$ orbitals govern the electronic structure of PCMs at $E_{\mathrm{F}}[25]$. Therefore, to reveal the influence of chemical disorder on the electronic properties of GST, we have analyzed the local density of these $p$ states (LDOS) on each atomic site. This quantity turns out to be mostly affected by the distribution of vacancies in the neighbouring shells. Since, for Te atoms, disorder occurs on the nearest-neighbour sites, whereas, for Ge and $\mathrm{Sb}$ atoms, it occurs on the next-nearest and third-nearest neighbour sites, the LDOS of Te atoms is much more sensitive to randomness. Therefore, in the following we focus on the Te states (for a discussion of the LDOS of Ge and Sb, see Supplementary Information).

Fig. 1 shows the LDOS averaged over all Te atoms having the same number of nearest-neighbour vacancies, $n_{\text {Vac }}$ : the LDOS in the vicinity of $E_{\mathrm{F}}$ increases drastically with $n_{\text {Vac }}$. For $n_{\text {Vac }}=0$, a minimum of the LDOS around $E_{\mathrm{F}}$ is observed. If, however, a vacancy is located near a Te atom, the corresponding Te $p$ orbital is shifted to higher energies, due to the reduced hybridization with neighbouring atoms. This shift is the larger, the more nearest neighbour vacancies are present around a Te atom. In particular for the configurations $n_{\text {Vac }}=3$ and 4 pronounced peaks appear slightly below or at $E_{\mathrm{F}}$. Therefore, the presence of neighbouring vacancies is shifting the Te states to higher energies near $E_{\mathrm{F}}$. To quantify this effect, in the inset of Fig. 1, the average LDOS at $E_{\mathrm{F}}$ is shown as a function of $n_{\text {Vac }}$, which clearly reveals the steep increase of the LDOS. Since the shift of the LDOS of a Te site depends almost exclusively on the number of vacancies in the nearest-neighbour shell, in a real space picture the LDOS can change drastically from one Te atom to the next. As a result, given a configuration $n_{\text {Vac }}=3$ or 4 , the hybridization of the Te $p$ states with states of neighbouring Te atoms is hindered. Hence, the $p$ states of the Te atoms located in regions having a high vacancy concentration (which we shall call vacancy clusters in the following) are expected to give rise to spatially localized electronic states near $E_{\mathrm{F}}$. The LDOS of the relaxed models shows similar trends (see Supplementary Information). The only significant effect of relaxation is to open a pseudo gap in the LDOS just above the Fermi en- 


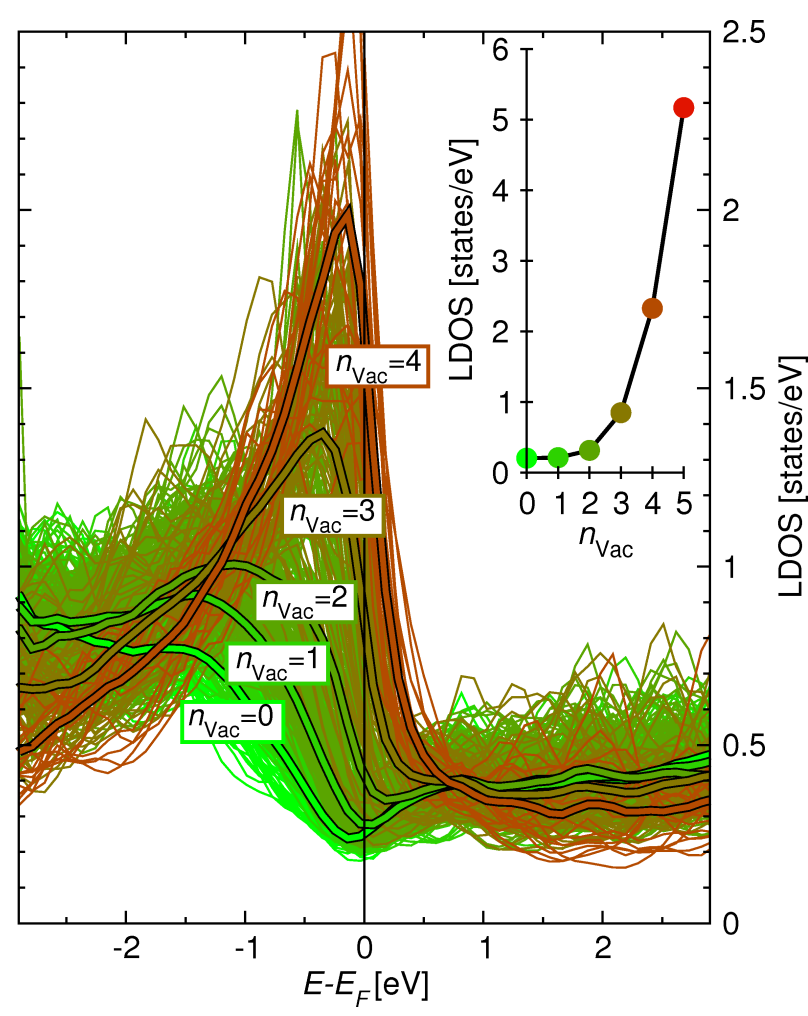

FIG. 1. Local density of states (LDOS) on the 500 Te sites in a $\mathrm{Ge}_{125} \mathrm{Sb}_{250} \mathrm{Te}_{500}$ supercell. Colour coding is used to differentiate between Te atoms with different number of nearest neighbour vacancies, $n_{\mathrm{Vac}}$. For each of these groups the average LDOS is shown as a thick line in the corresponding colour. An increasing number of nearest-neighbour vacancies leads to a pronounced increase in the density of Te states near $E_{\mathrm{F}}$. This is further corroborated in the inset, which shows the average LDOS at $E_{\mathrm{F}}$ on Te atoms as a function of $n_{\mathrm{Vac}}$, calculated from the bigger $\mathrm{Ge}_{512} \mathrm{Sb}_{1024} \mathrm{Te}_{2048}$ supercell.

ergy. The preceding paragraphs have provided convincing evidence that in the cubic phase a high density of Te $p$ states is created at $E_{\mathrm{F}}$ due to vacancies. These states are candidates for Anderson localization. Two crucial tasks still have to be tackled: (i) We need to show that electronic states are indeed localized at EF in c-GST. (ii) Furthermore, the structural rearrangement needs to be identified, which is responsible for the transition to the metallic state. We will address the latter question first. For this purpose, we have employed the wave function approach and considered a sequence of large models of GST containing 1008 atoms, starting from a Poisson distribution of $\mathrm{Ge}, \mathrm{Sb}$ and vacancies in c-GST. Subsequently, the amount of disorder in the system was reduced, until a perfectly ordered hexagonal phase was obtained, in the stacking sequence proposed in Ref. 26 (see Fig. 2 and the Supplementary Information). The latter phase is expected to form at very high annealing temperatures. All models considered were fully relaxed. Three important ingredients play a role in this sequence: the distribution of vacancies, the substitutional $\mathrm{Ge} / \mathrm{Sb}$ disorder and the arrangement of the crystalline planes (cubic or hexagonal). The sequence can be divided into two sets: in the first set, the distribution of vacancies becomes gradually more ordered, simulating the formation of vacancy layers in the system. This was accomplished by moving Ge and $\mathrm{Sb}$ atoms from every fourth layer to vacant sites in the other layers. This depletes the number of atoms in each fourth layer, until planes of vacancies are formed. After structural optimization, the distance between the Te layers at the two sides of a vacancy plane decreases significantly. Nevertheless, we continue referring to them as vacancy layers. For a fixed concentration of vacancies in the depleted layers, $l_{\text {Vac }}$, several configurations of vacancies, Ge and Sb atoms were considered, in both cubic and hexagonal arrangement. The black points in the plot of Fig. 2 indicate the energy per atom of the most stable structure for each $l_{V a c}$.

The rearrangement of vacancies affects the degree of compositional $\mathrm{Ge} / \mathrm{Sb}$ disorder as well, which is present in the structures of this set. In the final hexagonal model containing vacancy planes (denoted as $100 \%$ a in Fig. 2), two Ge/Sb layers out of three contain $75 \% \mathrm{Sb}$ and $25 \%$ $\mathrm{Ge}$, whereas the third layer contains $50 \% \mathrm{Sb}$ and $50 \%$ Ge. This arrangement corresponds to the model of hGST proposed in Ref. 27. Our results indicate that the hexagonal phase becomes lower in energy than the cubic phase for sufficiently large $l_{\text {Vac }}$ (above approximately 75 $\%)$. For these $l_{\text {Vac }}$, the energies of the most stable cubic models are also shown as red points in Fig. 2. Energy differences between cubic and hexagonal arrangements are relatively small, of the order of $5-7 \mathrm{meV}$ per atom. By far, the formation of the vacancy layers yields the most significant reduction in energy (of the order of 50 meV per atom). These findings suggest that the structural transition to the hexagonal phase is driven by the ordering of vacancies and takes place before the vacancy planes have completely formed.

In the second set (denoted as 100\% a-d in Fig. 2), all the models are hexagonal and contain vacancy planes. Substitutional disorder is reduced by swapping Ge and $\mathrm{Sb}$ atoms, until perfect $\mathrm{Ge}$ and Sb layers are formed, in the stacking sequence shown in the inset of Fig. $2(100 \%$ d). The latter phase is the lowest energy structure. However, energy differences between the models of this set are quite small, less than $5 \mathrm{meV}$ per atom, and are comparable to the configurational entropy contribution of the disordered phases. In summary, the atomic rearrangement which occurs upon annealing is characterized by the formation of vacancy layers, the decrease in compositional $\mathrm{Ge} / \mathrm{Sb}$ disorder and the change from cubic to hexagonal stacking. The ordering of vacancies leads to a large reduction in energy and also drives the change in stacking order.

Subsequently, we have investigated the electronic structure of these models in terms of the inverse par- 


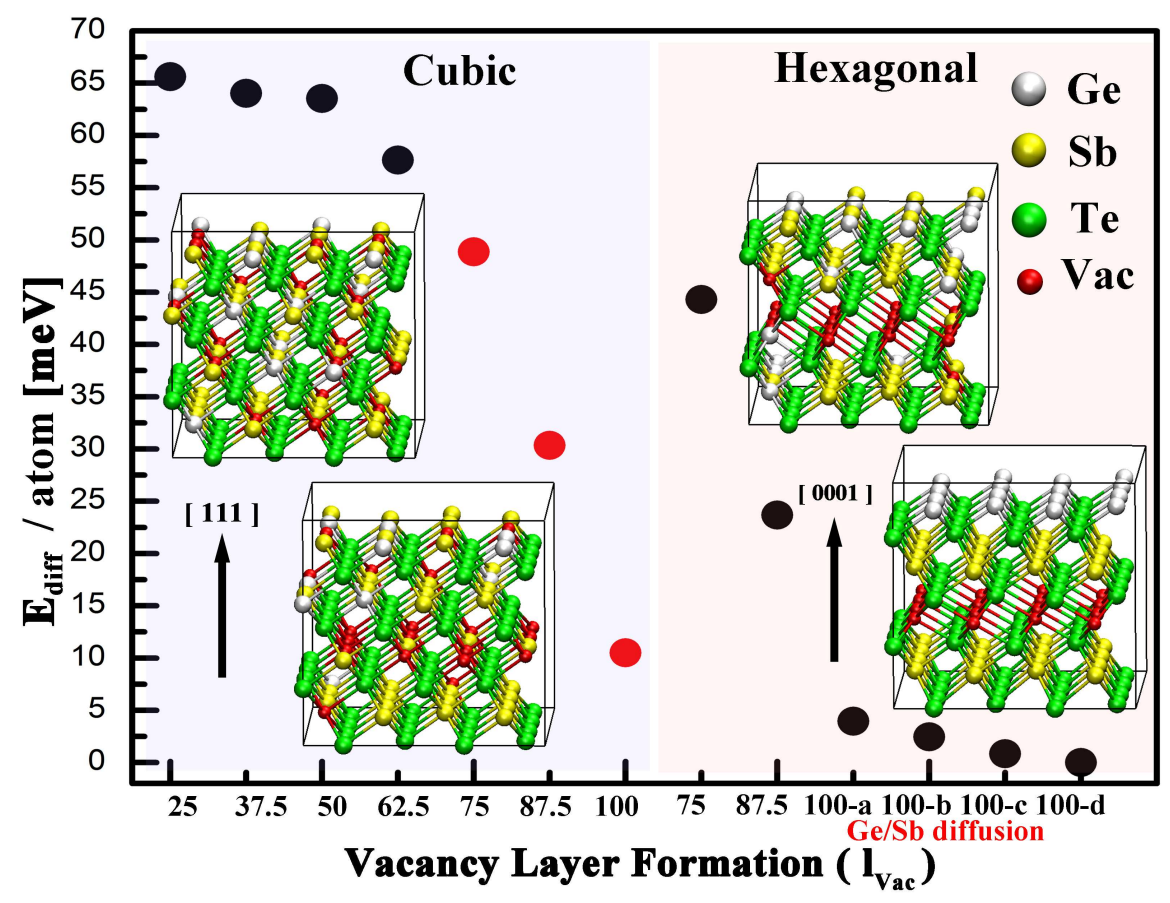

FIG. 2. a) Total energy per atom, $\mathrm{E}_{\text {diff }}$, of the models of cubic GST, hexagonal GST and intermediate structures studied. In the plot the zero of the energy coincides with the energy of the most favourable structure, hexagonal $100 \%$ d. Insets show the starting random cubic phase, the final "perfect" hexagonal phase and two intermediate phases. The random cubic phase contains 24 layers. In the structures where vacancy layers have fully formed, the number of atomic layers is 21 . The last four points $(100 \%$ a-d) correspond to hexagonal structures containing completely formed vacancy layers, which differ in the distribution of Ge and $\mathrm{Sb}$ atoms in the Ge/Sb layers. In particular, in models $100 \%$ a-c compositional Ge/Sb disorder is present on these layers. Model $100 \%$ a corresponds to the structure proposed in Ref. 27, which contains $75 \%$ Sb on the two outer $\mathrm{Ge} / \mathrm{Sb}$ layers and $50 \% \mathrm{Sb}$ on the central one (see Supplementary Information). The next two structures, b and c, are obtained by increasing the concentration of $\mathrm{Sb}$ on the outer Ge/Sb layers, and decreasing it on the central layer $(83 \%, 33 \%$ and $92 \%, 17 \%$ respectively). Finally, point d corresponds to the perfect hexagonal phase with two pure Sb layers and one pure Ge layer, as shown in the last inset. The plot clearly shows that the formation of the vacancy layers yields the most significant reduction in energy. It also suggests that the structural transition to the hexagonal phase occurs before the vacancy planes have completely formed.

ticipation ratios (IPRs) of the electronic states near $E_{\mathrm{F}}$ as shown in Fig. 3b. With the definition of the IPR, $\mathrm{IPR} \equiv \sum_{i}\left|\Psi_{\alpha, i}\right|^{4} / \sum_{i}\left(\left|\Psi_{\alpha, i}\right|^{2}\right)^{2}$, it is well suited to characterize the degree of localization of a Kohn-Sham eigenstate $\alpha$, where $\Psi_{\alpha, i}$ are the expansion coefficients of $\alpha$ with respect to the localized Gaussian-type orbitals (GTOs) forming the basis set (see Methods) and $i$ runs over all the GTOs. For an extended state, the IPR is equal to zero in infinitely large systems. On the contrary, for localized states, it remains finite and provides an estimate for the inverse of the number of atoms on which the state is localized. In the most disordered cubic phases, the IPR values of the states around $E_{\mathrm{F}}$ are of the order of $2-5 \cdot 10^{-2}$. These values are at least an order of magnitude larger than those of the extended states deep in the valence band. The latter values are below $2 \cdot 10^{-3}$, which reflects the fact that the number of atoms on which these extended states spread is of the same order of magnitude as the total number of atoms in the unit cell. These numbers explain why very large models are needed to unambiguously distinguish between localized and extended states. The calculated IPRs of $2-5 \cdot 10^{-2}$ show that, in disordered c-GST, states around $E_{\mathrm{F}}$ are localized in small regions of the crystals containing about 20-50 atoms. An example of such states is plotted in Fig. 3a. Inspection of the regions where these states are localized shows that they contain vacancy clusters, in agreement with our previous analysis. As an example, the vacancy concentration in the region within the localization radius of the state in Fig. 3a is about $200 \%$ larger than the average value (see Supplementary Material). This property holds both for the initial rocksalt structure and the intermediate phases. Assuming that the distribution of vacancies is Poissonian, vacancy clusters correspond to low-probability, local fluctuations of their concentration, in which the concentration exceeds its average value. These metastable configurations, although energetically unfavourable, are expected to occur at low annealing temperatures. At higher temperatures, these clusters disappear and vacancies form ordered two- 

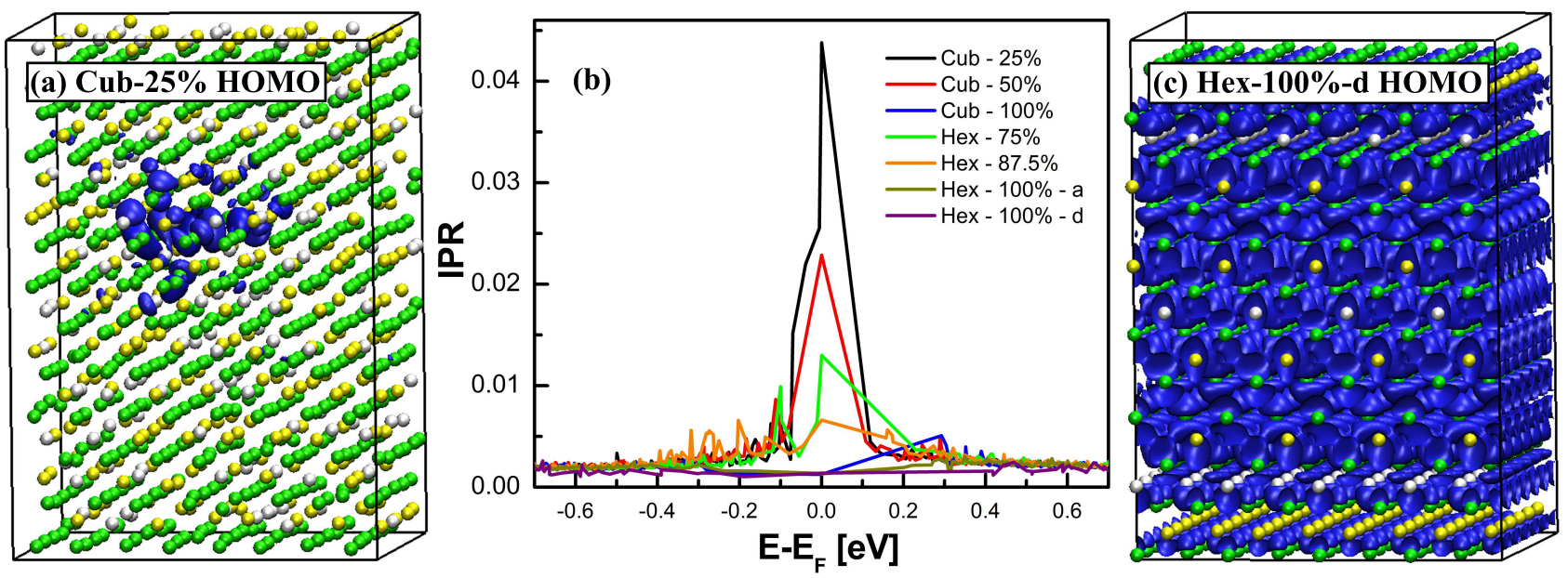

FIG. 3. a) Snapshot of the Highest Occupied Molecular Orbital (HOMO) of the completely random cubic model (Cub - $25 \%$ ) of GST. This state corresponds to the largest Inverse Participation Ratio (IPR) value $\left(4.4 \cdot 10^{-2}\right)$ shown in Fig. b. Isosurfaces render a value of 0.012 a.u: this value corresponds to the boundary region where the exponential decay of the state occurs (see Supplementary Information). b) IPR of the different models of cubic and hexagonal GST described in the text. Notations are the same as in Fig. 2. The dramatic decrease of the IPRs of states at $E_{\mathrm{F}}$ upon vacancy ordering provides clear evidence of a MIT driven by disorder. c) Snapshot of the HOMO state of the perfect hexagonal phase (Hex - $100 \%$ d) of GST. The corresponding IPR value is $1.4 \cdot 10^{-3}$

dimensional layers. This ordering brings about two effects. A gap of about $0.3-0.5 \mathrm{eV}$ opens up at $E_{\mathrm{F}}$ and the IPRs of the states near $E_{\mathrm{F}}$ decrease dramatically to values which are characteristic for delocalized wave functions (see Fig. 3). This second effect gives clear evidence of an MIT occurring in this system upon vacancy ordering. Compositional $\mathrm{Ge} / \mathrm{Sb}$ disorder, on the contrary, is insufficient to localize charge carriers near $E_{\mathrm{F}}$, as evidenced by the small values of the corresponding IPRs.

Transport measurements indicate that both phases have typically a relatively large density of carriers (in the range of $\left.1-2 \cdot 10^{20} \mathrm{~cm}^{-3}[15]\right)$. It is widely believed that these carriers are due to non-stoichiometric $\mathrm{Ge} / \mathrm{Sb}$ vacancies, which turn crystalline GST into a p-type degenerate semiconductor [28]. So far, such defects were not considered in our models. Since the concentrations of excess vacancies compatible with the experimental carrier densities are very small, inclusion of these defects in the disordered models does not shift the Fermi energy away from the region of localized states, as discussed in the Supplementary Information.

To summarize, the analysis of this set of models provides clear evidence for the occurrence of a structural transition and an Anderson MIT, both of them driven by vacancy ordering. It also indicates that the structural transition takes place well before the vacancy layers are completely formed. This shows that there is a significant amount of randomly distributed vacancies in h-GST close to this transition, which should yield localization of states at $E_{\mathrm{F}}$. These findings are compatible with experiments, which show that the MIT occurs in the hexagonal phase [15]. Nevertheless, although the two transitions have been shown to occur almost simultaneously in our models, the phenomena are, in principle, independent. Thus it would be interesting to "disentangle" the effects of the two transitions. For this purpose, it is useful to further analyze the complete sequence of cubic models of GST shown in Fig. 2, in which Ge and $\mathrm{Sb}$ atoms are moved until vacancy layers are formed but no rearrangement of the planes from cubic to hexagonal stacking occurs. Fig. 3b shows that the IPRs near $E_{\mathrm{F}}$ decrease dramatically upon vacancy ordering in this set as well, thus demonstrating that the cubic-hexagonal transition is not relevant for the occurrence of the MIT.

In conclusion, we have shown that the MIT recently observed in crystalline GST is due to the decrease of randomness brought about by the ordering of vacancies. We have identified the localization of the spatially directed Te $p$ electrons by relatively small clusters of randomly distributed vacancies on the $\mathrm{Ge} / \mathrm{Sb}$ sublattice as the origin of the high resistance. Substitutional disorder and structural distortions do not play a crucial role in this MIT. Similar properties are expected to hold for other PCMs along the $\mathrm{GeTe}-\mathrm{Sb}_{2} \mathrm{Te}_{3}$ line containing a large number of intrinsic vacancies, such as the technologically important $\mathrm{Ge}_{2} \mathrm{Sb}_{2} \mathrm{Te}_{5}$ alloy. These properties make these systems unique: in doped semiconductors, a MIT is induced by increasing the concentration of extrinsic defects; on the contrary, in PCMs a large number of intrinsic defects is present and the MIT is solely induced by their rearrangement. These findings, together with the fact that vacancy ordering is easier to induce and control than com- 
positional $\mathrm{Ge} / \mathrm{Sb}$ disorder, due to the large reduction of energy involved in the former process, open up the possibility of tuning the resistance of phase-change devices in a controlled and reproducible fashion. We conjecture that in materials with $p$ electrons at the Fermi energy a localization of their wavefunctions by small vacancy clusters should be a general mechanism. Considering that in compounds with vacancy layers, such as the transitionmetal dichalcogenides, the vacancy distribution may be controlled similar to GST, we expect a large class of materials exhibiting an MIT due to vacancy ordering.

\section{METHODS}

Density Functional Theory calculations: the calculations of the two models of rocksalt GST containing 900 atoms and 3584 atoms, $\mathrm{Ge}_{125} \mathrm{Sb}_{250} \mathrm{Te}_{500}$ and $\mathrm{Ge}_{512} \mathrm{Sb}_{1024} \mathrm{Te}_{2048}$, were done using KKRNano, a massively parallelized code based on an all-electron, full-potential Korringa-Kohn-Rostoker Green function method [29]. A cubic supercell and the experimental lattice constant of $6.04 \AA$ were used. In these calculations empty cells were included on vacancy sites to describe the lattice accurately. We used an angular momentum cut-off of $l_{\max }=3$ and the $\Gamma$-point to sample the Brillouin zone. The calculations were performed in the (scalar-relativistic) local spin density approximation (LSDA) [30]. Relaxation of the big models described in the second part of the paper was done using QUICKSTEP [31], a code based on a mixed Gaussian and plane-wave approach, included in the CP2K suite of programs [32]. The Kohn-Sham orbitals were expanded in a Gaussian-type basis set of triple-zeta plus polarization quality, whereas the charge density was expanded in plane waves, with a cutoff of 300 Ry. Goedecker [33] pseudopotentials and gradient-corrected functionals [34] were used in these simulations. Elongated supercells containing 24 atomic layers along the $z$ direction (21 layers for the models containing fully formed vacancy planes) were used: this large number of layers is needed to correctly describe the hexagonal phase. The energies plotted in Fig. 2 correspond to the models with experimental lattice constants: relaxation of the cells was shown not to change the relative energetics of the cubic and hexagonal models.

* Email address: wuttig@physik.rwth-aachen.de

$\dagger$ Email address: mazzarello@physik.rwth-aachen.de

[1] Anderson, P. W. Absence of diffusion in certain random lattices. Phys. Rev. 109, 1492-1505 (1958).

[2] Mott, N. F. Conduction in glasses containing transition metal ions. J. Non-Cryst. Solids 1, 1-17 (1968).
[3] Alexander, M. N. \& Holcomb, D. F. Semiconductor-tometal transition in $n$-type group IV semiconductors. Rev. Mod. Phys. 40, 815-829 (1968).

[4] Rosenbaum, T. F., Andres, K., Thomas, G. A. \& Bhatt, R. N. Sharp insulator transition in a random solid. Phys. Rev. Lett. 45, 1723-1726 (1980).

[5] Gaymann, A., Geserich, H. P. \& Löhneysen, H. V. Temperature dependence of the far-infrared reflectance spectra of Si:P near the metal-insulator transition. Phys. Rev. B 52, 16486-16493 (1995).

[6] Kramer, B. \& MacKinnon A. Localization: theory and experiment. Rep. Prog. Phys. 56, 1469-1564 (1993).

[7] Abrahams, E., Anderson, P. W., Licciardello, D. C. \& Ramakrishnan, T. V. Scaling theory of localization: Absence of quantum diffusion in two dimensions. Phys. Rev. Lett. 42, 673-676 (1979).

[8] Wegner, F. The mobility edge problem: Continuous symmetry and a conjecture. Z. Phys. B 35, 207-210 (1979).

[9] Shklovskii, B. I. \& Efros, A. L. Electronic Properties of Doped Semiconductors (Springer, 1984).

[10] Efetov, K. B. Supersymmetry in disorder and chaos (Cambridge University Press, 1997).

[11] Hubbard, J. Electron Correlations in Narrow Energy Bands. Proc. R. Soc. London A 276, 238 (1963).

[12] Georges, A. \& Kotliar, G. Hubbard model in infinite dimensions. Phys. Rev. B 45, 6479 (1992).

[13] Kotliar, G. et al.. Electronic structure calculations with dynamical mean-field theory. Rev. Mod. Phys. 78, 865 (2006).

[14] Dong, J. \& Drabold, D. A. Atomistic Structure of BandTail States in Amorphous Silicon. Phys. Rev. Lett. 80, 1928-1931 (1998).

[15] Siegrist, T. et al. Disorder-induced localization in crystalline phase-change materials. Nature Mater. 10, 202208 (2011).

[16] Shportko, K. et al.. Resonant bonding in crystalline phase-change materials. Nature Mater. 7, 653-658 (2008).

[17] Lencer, D. et al.. A map for phase-change materials. Nature Mater. 7, 972-977 (2008).

[18] Peierls, R. E. Quantum Theory of Solids (Oxford University Press, 1956).

[19] Wuttig, M. et al.. The role of vacancies and local distortions in the design of new phase-change materials. Nature Mater. 6, 122-128 (2007).

[20] Wuttig, M. \& Yamada, N. Phase-change materials for rewriteable data storage. Nature Mater. 6, 824-832 (2007).

[21] Korringa J. On the calculation of the energy of a Bloch wave in a metal. Physica 13, 392 (1947).

[22] Kohn, W. \& Rostoker, N. Solution of the Schrödinger equation in periodic lattices with an application to metallic Lithium. Phys. Rev. 94, 1111 (1954).

[23] Raoux, S. \& Wuttig, M. (Ed.) Phase Change Materials: Science and Applications (Springer, 2008).

[24] Yamada, N. \& Matsunaga, T. Structure of lasercrystallized $\mathrm{Ge}_{2} \mathrm{Sb}_{2+x} \mathrm{Te}_{5}$ sputtered thin films for use in optical memory. J. Appl. Phys. 88, 7020-7028 (2000).

[25] Wełnic, W. et al. Unravelling the interplay of local structure and physical properties in phase-change materials. Nature Mater. 5, 56-62 (2005).

[26] Kooi, B. J. \& De Hosson, T. M. J. Electron diffraction and high-resolution transmission electron microscopy of the high temperature crystal structures of GexSb2Te3+x $(\mathrm{x}=1,2,3)$ phase change material. J. Appl. Phys. 92, 
3584-3590 (2002).

[27] Matsunaga, T. \& Yamada, N. Structural investigation of $\mathrm{GeSb}_{2} \mathrm{Te}_{4}$ : A high-speed phase-change material. Phys. Rev. B 69, 104111 (2004).

[28] Lee, B.-S. et al. Investigation of the optical and electronic properties of $\mathrm{Ge}_{2} \mathrm{Sb}_{2} \mathrm{Te}_{5}$ phase change material in its amorphous, cubic, and hexagonal phases. J. Appl. Phys. 97, 093509 (2005).

[29] Thiess, A., Zeller, R., Bolten, M., Dederichs, P. H. \& Blügel, S. Massively parallel density functional calculations for thousands of atoms: KKRnano. Submitted to Phys. Rev. B.

[30] von Barth, U. \& Hedin, L. A local exchange-correlation potential for the spin polarized case: I. J. Phys. C 5, 1629-1642 (1972).

[31] VandeVondele, J. et al. QUICKSTEP: Fast and accurate density functional calculations using a mixed Gaussian and plane waves approach. Comput. Phys. Commun. 167, 103-128 (2005).

[32] Krack, M. \& Parrinello, M. in High Performance Computing in Chemistry Vol. 25 (ed. Grotendorst, J.) 29-51 (NIC, 2004); http://cp2k.berlios.de.

[33] Goedecker, S., Teter, M. \& Hutter, J. Separable dualspace Gaussian pseudopotentials. Phys. Rev. B 54, 17031710 (1996).

[34] Perdew, J. P., Burke, K., Ernzerhof, M. Generalized gradient approximation made simple. Phys. Rev. Lett. 77, 3865-3868 (1996).

\section{ACKNOWLEDGEMENTS}

We thank S. Caravati and Y. Li for useful discussions. We gratefully acknowledge funding by the DFG (German Science Foundation) within the collaborative research centre SFB 917 "Nanoswitches", as well as the computational resources by the RWTH Rechenzentrum and the Forschungszentrum Jülich.

\section{AUTHOR CONTRIBUTIONS}

W. Z. and A. T. performed the CP2K and KKRNano simulations respectively. Analysis of the data was mostly carried out by W. Z. and R. M. (CP2K) and A. T., R. Z. and P. H. D. (KKRNano). The paper was written by R. M. and M. W., with help from all co-authors, in particular W. Z. and A. T. The project was initiated and conceptualized by S. B., R. M. and M. W.

\section{ADDITIONAL INFORMATION}

The authors declare no competing financial interests. Supplementary information accompanies this paper on www.nature.com/naturematerials. Reprints and permissions information is available online at http://npg.nature.com/reprintsandpermissions. Correspondence and requests for materials should be addressed to R. M. or M. W. 